\title{
Non-linear laminar flow of fluid through a vertically stratified cylindrical porous aquifer
}

\author{
S. K. J J AIN $\left(^{*}\right)$ \\ Received on May 24th, 1982
}

\begin{abstract}
This note presents the study of non-linear laminar flow of fluid through a vertically strafified cylindrical porous aquifer in the steady state condition. The influence of non-linear laminar flow on the discharge of the fluid and its dependence on the related physical quantities, is discussed. In particular, results for non-linear laminar flow of fluid into a concentric well fully penetrating the homogeneous aquifer have been deduced and compared.
\end{abstract}

\section{Riassunto}

La presente nota riguarda lo studio del flusso laminare non-lineare di un fluido at traverso un "aquifer" verticalmente stratificato poroso in condizioni stazionarie.

L'influenza del flusso laminare non-lineare sul processo di discarica viene discussa con la sua dipendenza dalle quantità fisiche correlate. In particolare vengono dedotti e comparati i risultati per il flusso non laminare del fluido in un pozzo concentrico penetrante l'aquiter omogeneo.

( $\left.{ }^{\star}\right)$ Department of Mathematics, Rivers State College of Education, Rumuolumeni, P. M. B. 5047, PORT HARCOURT (NIGERIA). 


\section{1. - INTRODUCTION}

The nature of flow mainly depends upon the fluid velocity and the structural constitution of the porous matrix through which it flows. On the basis of fluid velocity, however, the flow (Bear, 1972) can be characterized into three different regimes - laminar, non-linear laminar and turbulent, Reynolds number being the criteria for such demarcation. But, the intricacy of the nature of porous media does not always justify the natural flow of fluid to be purely laminar and it appears more desirable to be either non-linear laminar or turbulent. Consequently, Uchida, 1952, Engelund, 1953, Anandkrishan and Varadarajulu (1963), Whright, 1968, Ahmad and Sunada (1969), Khan and Raza (1972), Jain and Upadhyay (1976), Upadhyay (1975, 1977) and others obtained specific solutions of certain non-linear flow problems.

In the present paper, we consider non-linear laminar steady state flow of fluid into an uncased well, concentrically established with respect to the contour of intake and penetrating fully the vertically stratified porous aquifer of finite thickness. It is found that the flow pattern is characterized by two different zones, in which the discharge exhibits opposite character as regards its dependence on grain size of the medium, viscosity of the fluid and radius of the well, is concerned. Further, it is observed that as the permeability of the region in the vicinity of the well decreases or as the region becomes narrower, in both the situations, the influence of non-linear laminar flow is to increase the discharge.

As a particular case, the results for non-linear laminar flow of fluid into a concentric well fully penetrating the homogeneous aquifer have been deduced and compared with those obtained by Upadhyay, 1977.

\section{2. - EQUATIONS OF FLUID FLOW IN POROUS MEDIUM}

The laws governing laminar and non-linear laminar flow of fluid in porous media are (Poluvarinova-Kochina, 1962).

$$
v=-k \frac{\mathrm{d} h}{\mathrm{~d} s}
$$


and

$$
\frac{\mathrm{d} h}{\mathrm{~d} s}=a v+b v^{2}
$$

where $v, k, \frac{\mathrm{d} h}{\mathrm{~d} s}$ denote seepage velocity, seepage coefficient and hydraulic gradient respectively; constants $a$ and $b$, according to Engelund (1953) are

$$
\frac{a}{p}=\frac{2000 \mu}{g d^{2}}, b=\frac{35}{g d}
$$

$\rho$ and $\mu$ being density and viscosity of the fluid respectively and $d$ the grain size of the medium.

Beside relations [1] and [2], we consider the expression for pressure distribution in the case of a concentrically placed circular well fully penetrating a two-layered vertically stratified cylindrical porous aquifer of thickness $T$ in the form (Poluvarinova-Kochina, 1962)

$$
p=\frac{Q \mu}{2 \pi k_{1} T}\left[\log \left(\frac{r_{0}}{r_{\omega}}\right)+\frac{l}{k_{2}} \log \left(\frac{r}{r_{0}}\right)\right]+C
$$

where $C$ is the constant, to be determined with the help of boundary conditions consistent with the system.

\section{3. - Statement of the Problem}

In the steady state condition, we consider the flow of fluid into an uncased well of radius $r_{\omega}$, concentrically established with respect to the contour of intake of radius $r_{\varepsilon}$. It is assumed that the well is fully penetrating the porous aquifer of thickness $T$. The aquifer is considered to be vertically stratified into adjacent cylindrical regions of permeabilities $k_{1}$ and $k_{2}$ separated by the boundary $r=r_{0}$. The pressure at the contour of well and that of intake are prescribed as $p_{\mathrm{s}}$ and $p_{c}$ respectively. 
In the present system, we assume the flow to be non-linear laminar $r_{t}$ within i narrow cylindrical zone $r_{\omega}>r \leqslant r_{t}$ and laminar in the region $r_{t}<r \leqslant r_{c}$. Hence it becames obvious from physical considerations that $k_{1} \geqslant k_{2}$. Let $p_{t}$ be the pressure at the transition boundary $r=r_{t}$ [cf. fig. 1 nel testo].

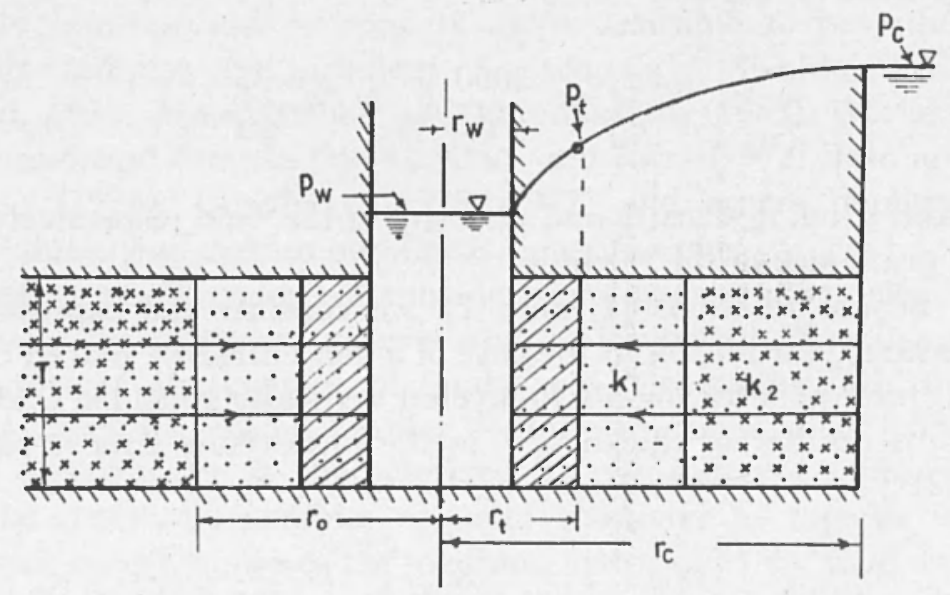

Fig. 1

The problem is to examine the influence of non-linear laminar flow on the discharge of fluid and its dependence on the related physical quantities.

\section{4. - Solution}

For laminar zone, the boundary conditions are

$$
\begin{aligned}
& p=p_{c} \text { at } r=r_{c}, \\
& p=p_{t} \text { at } r=r_{t}
\end{aligned}
$$


which on substitution in [4] gives

$$
p_{t}=p_{c}+\frac{Q \mu}{2 \pi k_{1} T}\left[\frac{k_{1}}{k_{2}} \log \left(\frac{r_{t}}{r_{s}}\right)\right]
$$

As $p=\rho g h$ and $Q=2 \pi r I v$, equation [2] becomes

$$
\frac{d p}{d r}=\rho_{y}\left[\frac{a Q}{2 \pi r T}+\frac{b Q^{2}}{4 \pi^{2} r^{2} T^{2}}\right]
$$

Integrating [7] and then evaluating the constant of integration with the help of boundary conditions

$$
\begin{aligned}
& p=p_{\omega} \text { at } r=r_{\omega}, \\
& p=p_{t} \text { at } r=r_{t \prime}
\end{aligned}
$$

we obtain

$p_{r}=p_{w}+\rho g\left[\frac{a Q}{2 \pi T} \log \left(\frac{r_{t}}{r_{\omega}}\right)=\frac{b Q^{2}}{4 \pi^{2} T^{2}}\left(\frac{1}{r_{\omega}}-\frac{1}{r_{t}}\right)\right]$

At the boundary of transition from laminar to non-linear laminar flow, the relation between critical Reynolds number $\xi_{c}=0.07$ and critical velocity $v_{c}$ is given by

$$
v_{c}=\frac{Q}{2 \pi r_{t} T}=\frac{a}{b} \check{\zeta}_{c}
$$

Since at the boundary of transition $\frac{\mathrm{d} h}{\mathrm{~d} s}$ as given by [1] and [2] yield the same value, it follows from [10] that

$$
1.07 a k_{1} \rho g=\mu
$$

Using [11] in [6] and then equating the value of $p$, so obtained with that given by [9], we get 


$$
\begin{aligned}
& \frac{p_{c}-p_{\omega}}{P g}=\frac{a Q}{2 \pi T}\left[\log \left(\frac{r_{t}}{r_{\omega}}\right)-1.07 \frac{k_{l}}{k_{2}}\right. \\
& \left.\log \left(\frac{r_{t}}{r_{c}}\right)\right]+\frac{b Q^{2}}{4 \pi^{2} T^{2}}\left(\frac{1}{r_{\omega}}-\frac{1}{r_{t}}\right)
\end{aligned}
$$

Combining equations $[3 \mathrm{a}, \mathrm{b}]$ and $[10]$ with $[12]$, we obtain

$$
\begin{gathered}
\frac{\rho d^{3}\left(p_{c}-p_{\omega}\right)}{\mu^{2} r_{\omega}}=8000 \frac{r_{t}}{r_{\omega}}\left[\log \left(\frac{r_{t}}{r_{\omega}}\right)-1.07\right. \\
\left.\frac{k_{l}}{k_{2}} \log \left(\frac{r_{c}}{r_{c}}\right)+0.07\left(\frac{r_{t}}{r_{\omega}}-1\right)\right]
\end{gathered}
$$

If we assume the flow of fluid to be purely laminar in the entire region $r_{\omega} \leqslant r \leqslant r_{c}$, then the flow rate $Q_{\text {lam }}$ may be obtained from [4] by using the corresponding boundary conditions both at the contour of intake and at the well. Thus

$$
Q_{\text {lam }}=\frac{2 \pi k_{1} T}{\mu} \quad\left\{\frac{\left(p_{c}-p_{\omega}\right)}{\log \left(\frac{r_{0}}{r_{\omega}}\right)+\frac{k_{1}}{k_{2}}, \log \left(\frac{r_{c}}{r_{o}}\right)}\right\}
$$

and therefore, using [10] and [14], we obtain

$$
\begin{array}{r}
\left.\frac{Q}{Q_{\text {lam }}}=8560 \frac{r_{i}}{{ }^{\prime} \omega} \mid \frac{\mu^{2} r_{\omega}}{\rho d^{3}\left(p_{c}-p_{\text {s. }}\right)}\right\} \\
{\left[\log \left(\frac{r_{o}}{r_{\omega}}\right)+\frac{k_{1}}{k_{2}} \log \left(\frac{r_{\mathrm{c}}}{r_{o}}\right)\right]}
\end{array}
$$

Introducing dimensionless quantity $X$ and ratio $Y$ such that

$$
X=\frac{P d^{3}\left(p_{c}-p_{\omega}\right)}{\mu^{2} r_{\omega}} . \quad Y=\frac{Q}{C_{\text {llam }}},
$$

and combining [15] with [13], we obtain an implicit relation 
$1.07 X=\frac{X Y}{Z}\left[\left(1-1.07 \frac{k_{\mathrm{i}}}{k_{2}}\right) \log \left(\frac{X Y}{8560 Z}\right)+\right.$

$\left.1.07 \frac{k_{1}}{k_{2}} \log \left(\frac{r_{c}}{r_{t 1}}\right)+0.07 \frac{X Y}{8560 Z}-0.07\right]$

where

$$
Z=\left[\log \left(\frac{r_{0}}{r_{\omega}}\right)+\frac{k_{1}}{k_{2}} \log \left(\frac{r_{c}}{r_{0}}\right)\right]
$$

In view of physical considerations it may, however, be inferred from [16 a] that $X>0$, hence equation [17] becomes

$$
\begin{aligned}
& 1.07 Z=Y\left[\left(1-1.07 \frac{k_{1}}{k_{2}}\right) \log \left(\frac{X Y}{8560 Z}\right)+\right. \\
& \left.1.07 \frac{k_{1}}{k_{2}} \log \left(\frac{r_{c}}{r_{\omega}}\right)+0.07 \frac{X Y}{8560 Z}-0.07\right]
\end{aligned}
$$

\section{5. - Particular case}

If $k_{1}=k_{2}$, that is, the entire flow region is homogeneous with uniform permeability, equation [18] reduces to

$$
\begin{gathered}
1.07 \log \left(\frac{r_{c}}{r_{\omega}}\right)=Y\left[1.07 \log \left(\frac{r_{c}}{r_{\omega}}\right)+0.07\right. \\
\left.\frac{X Y}{8560 \log \left(\frac{r_{c}}{r_{\omega}}\right)}-0.07 \log \left\{\frac{X Y}{8560 \log \left(\frac{r_{c}}{r_{\omega}}\right)}\right\}-0.07\right],
\end{gathered}
$$

which represents the non-linear laminar flow of fluid into a concentric well fully penetrating the homogeneous porous aquifer discussed by Upadhyay (1977). 


\section{6. - Discussion}

From [16a] it is evident that $X$ depends on the density of fluid, grain size of the medium, pressure difference of the system, viscosity of the fluid and well radius. Since $d$ and $\mu$ occur in higher powers in the expression for $X$, they highly affect the discharge.

In case of non-linear laminar flow of fluid, the inertial forces are predominant which causes more resistance and consequently smaller discharge that might be expected in laminar flow. Obviously the effect of non-linear laminar flow can be observed only when $Y<1$.

To get a definite idea of result [18], we take the system for which $r_{c}=3 \times 10^{3} r$, that is, the radius of contour of intake is equal to 3000 times the radius of the well. Assuming $r_{0}=1.5 \times 10^{3} \mathrm{r}$, we analyse the effect of permeabilities of the stratified zones on the discharge, in the particular cases.

$$
k_{1}=3 k_{2}, 2 k_{2}, k_{2} \text {. }
$$

The first two of which imply that the permeability of the inner zone is respectively 3 and 2 times that of the outer zone, where as the last value corresponds to the case of uniform permeability.

Using [18] and [20], the numerical values of $Y$ are obtained in Table I corresponding to different values of $X>0$.

TABLE I

Values of $\left.Y ; r_{0}=1.50 \times 10^{3} \times r_{(}\right)$

\begin{tabular}{ccccccccccc}
\hline$k_{1} / k_{2} X$ & $\ldots \ldots$ & 10 & $10^{2}$ & $10^{3}$ & $10^{4}$ & $10^{5}$ & $10^{6}$ & $10^{7}$ & $10^{8}$ & $10^{9}$ \\
& & & & & & & & & & \\
3.0 & $\ldots \ldots \ldots$ & 0.2028 & 0.2320 & 0.2596 & 0.3061 & 0.3612 & 0.4778 & 0.4835 & 0.2712 & 0.1018 \\
2.0 & $\ldots \ldots \ldots$ & 0.2952 & 0.3217 & 0.4045 & 0.4450 & 0.5212 & 0.6111 & 0.5396 & 0.3650 & 0.0908 \\
1.0 & $\ldots \ldots \ldots$ & 0.9394 & 0.9561 & 0.9739 & 0.9912 & 0.9994 & 0.9253 & 0.5988 & 0.2526 & 0.0876 \\
\hline
\end{tabular}

These tabulated values have been graphically represented in Fig. 2, abscissa and ordinate being $X$ and $Y$ respectively. It is seen 


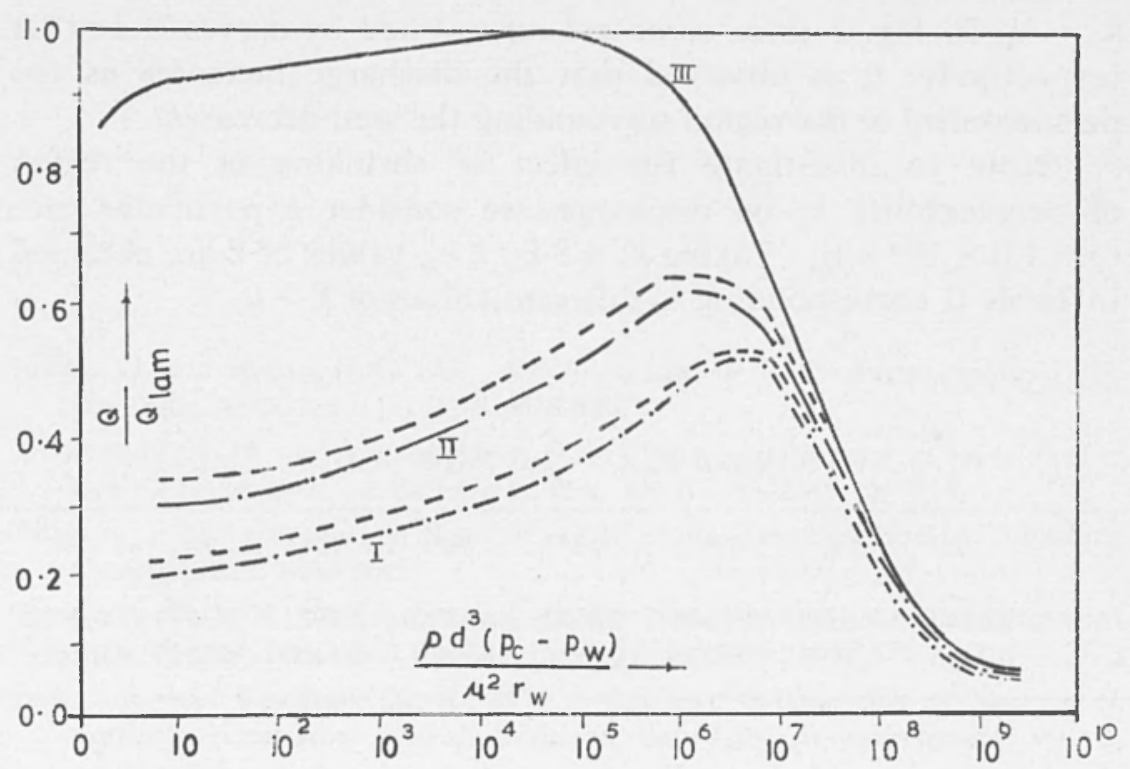

Fig. 2

from Fig. 2 that in curve $-I$ which corresponds to $k_{1}=3 k_{2}$, as $X$ increases, initially $Y$ increases till it attains a maximum value 0.4970 (corresponding to $X=4.85 \times 10^{6}$ ); afterwards it decreases asymptotically. Thus, in the former region $0<X \leqslant 4.85 \times 10^{6}$ the discharge increases as $X$ increases, that is, when the density of the fluid, grain size of the medium and pressure difference of the system increases, viscosity of the fluid and well radius decreases. In the later region $X>4.85 \times 10^{6}$ the influence of non-linear laminar flow is reversed.

Hence, it may be concluded that in case of non-linear laminar flow, the flow pattern is characterised by two different zones in which discharge exhibits opposite character.

\section{7. - COMPARISION}

To examine how the permeabilities of the stratified zones affect to discharge, we consider $k_{1}=2 k_{2}$ and the limitirig case 
$k_{1}=k_{2}$. In Fig. 2 these cases are represented by curves II and III respectively. It is observed that the discharge increases as the permeability of the region surrounding the well decreases.

Now to investigate the effect of shrinking of the region of permeability $k_{1}$ on discharge, we consider a particular case $r_{0}=1.0 \times 10^{3} \times r_{\omega}$. Taking $k_{1}=3 k_{2}, 2 k_{2}$, values of $Y$ are obtained in Table II corresponding to different values of $X>0$.

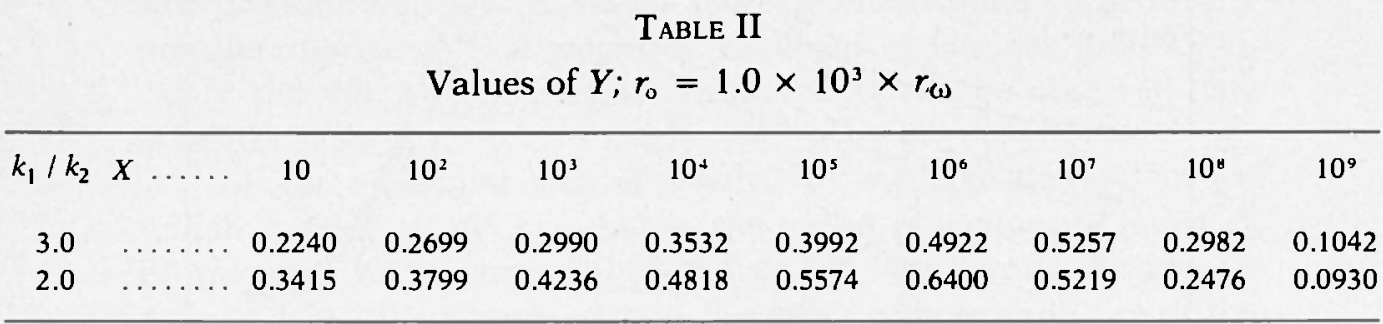

These values have been graphically shown in Fig. 2 by dotted curves. It is inferred that discharge also increases when the region of permeability $k_{1}$ becomes narrower.

Hence, in general it may be concluded that the shrinking of the zone surrounding the well, as well as decrease, in its permeability causes a larger discharge comparatively, which is an obvious physical phenomenon. 


\section{REFERENCES}

AHmad, N. and SunADa, D.K., 1969 - Non-linear laminar flow in porous media. " J. Hy. Dn., Proc. ASCE ", 95, HY6, pp. 1847-1857.

ANANDKRishan, M. and Varadarajulu, G.H., 1963 - Laminar and turbulent flow of water through sand. "J. Soil Mech., Proc. ASCE », 89, SM5, pp. 1-15.

BEAR. J., 1972 - Dynamics of fluids in porous media. American Elsevier Publishing Company Inc., New York.

ENGELUND, F., 1953 - On laminar and turbulent flow of ground water in homogeneous sand. "Trans. Dan. Acad. Tech. Sci.», 3, pp. 1-105.

JAIN, S.K. and UPADHYAY, K. K., 1976 - Non-linear laminar flow of fluid into a partially penetrating well. "Gerlands Beiträge Zur Geophysik», 85, 2 . pp. 151-156.

KEAN, I.H. and RAZA, S.A., 1972 - A study of non-linear flow through porous media by electrical analogy method. " J. Inst. of Engg. India, C.E. Dn. », 53, pp. 57-60.

Polubarinova - Kochina, P.Ya., 1962 - Theory of ground water movement. Princeton University Press, Princeton, New Jersey.

UchIDA, S., 1925 - On the non-linear parcolation at high Reynolds number. "Nat. Committee for Theor. Appl. Mech.», pp. 437-442.

UPADHYAY, K.K., 1975 - Non-linear laminar flow into eccentrically placed well. «Annali di Geofisica », XXVIII, 4, pp. 311-319.

UPADHYAY, K.K., 1977 - Non-linear laminar flow into well partielly penetrating a porous aquifer of finite thickness. "Pure and Applied Geophysics», 115, pp. 631-638.

UPADHYAY, K.K., 1977 - Non-linear laminar flow of fluid into a fully penetrating cylindrical well. «Ind. Jour. Theor. Physics», 25, N. 4, pp. 149-156.

WRIGHT, D.E., 1968 - Non-linear flow through grannular media. «J. Hy. Dn., Proc. ASCE $n, 94$, HY 4, pp. 851-872. 